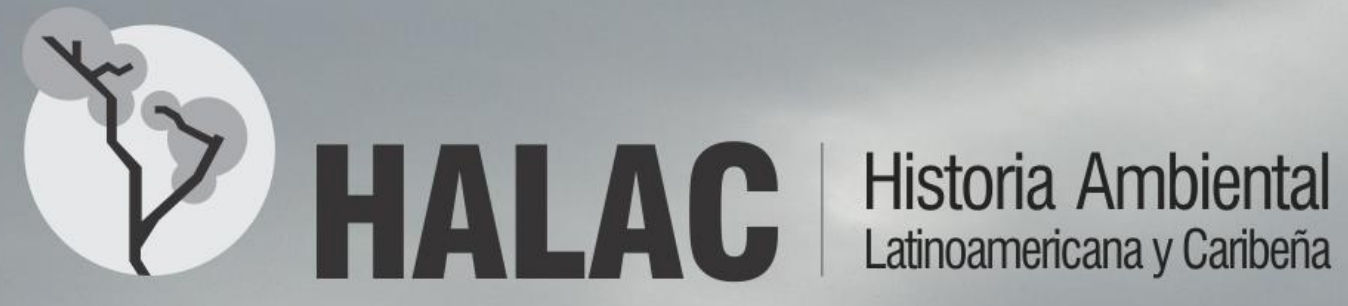

Volumen IX, Número 2 (2019)

\title{
Balances de Historia Ambiental en América Latina
}




\section{is \\ HALAC}

JUNTA DIRECTIVA SOLCHA 2016-2018

\section{Presidente}

Wilson Picado, Universidad Nacional (Costa Rica)

\section{Vicepresidente}

Nicolás Cuvi, Facultad Latinoamericana de Ciencias Sociales (Ecuador)

\section{Secretaria}

Aceneth Perafán Cabrera, Universidad del Valle (Colombia)

\section{Editor General de la Revista HALAC}

Sandro Dutra e Silva, Universidade Estadual de Goiás (Brasil) | Centro Universitário de Anápolis (Brasil)

Marina Miraglia, Universidad Nacional de General Sarmiento (Argentina)

\section{Encargado de Comunicaciones}

Chris Boyer, University of Illinois at Chicago (EEUU)

\section{Encargados de la Página Web}

Micheline Cariño, Universidad Autónoma de Baja California Sur (México)

Antonio Ortega Santos, Universidad de Granada (España)

\section{Encargada de la Bibliografía en Línea}

Ana Marcela França, Universidade Federal do Rio de Janeiro, UFRJ, Brasil. Ayelen Dichdji, Universidad Nacional de Quilmes, Argentina. 


\section{is \\ HALAC}

\section{Coordinación Editorial}

Dr. Sandro Dutra e Silva, Universidade Estual de Goiás, Brasil | Centro Universitário de Anápolis, Brasil | Editor

Dr. Marina Miraglia, Universidad Nacional de General Sarmiento, Argentina |

Editora

\section{Editores de Reseñas}

Dr. Matthew Vitz, University of California, San Diego, United States

Dr. Samira Peruchi Moretto, Universidade Federal da Fronteira Sul, Brasil

Dr.Vladimir Sánchez Calderón, Universidad Industrial de Santander, Colômbia

\section{Consejo Editorial}

Dr. Adrián Zarrilli, Centro de Estudios de la Argentina Rural | Departamento de Ciencias Sociales | Universidad Nacional de Quilmes | Buenos Aires, Argentina

Dr. Fernando Ramirez Morales, Universidad de Chile | Santiago, Chile

Dr. Jo Klanovicz, Universidade Estadual do Centro Oeste (Unicentro) | Programa de Pós Graduação em História | Programa de Pós Graduação Interdisciplinar em Desenvolvimento Comunitário | Guarapuava | Paraná, Brasil

Dr. Lise Fernanda Sedrez, Universidade Federal do Rio de Janeiro (UFRJ) | Programa de Pós Graduação em História Social | Instituto de História, Brasil

Dr. Patricia Clare, Universidad de Costa Rica | Escuela de Historia | San José, Costa Rica

Dr. Regina Horta Duarte, Universidade Federal de Minas Gerais | Programa de Pós Graduação em História | Faculdade de Filosofia e Ciências Humanas | Belo Horizonte | Minas Gerais, Brasil

Dr. Reinaldo Fuñes Monzote, Universidad de La Habana | Departamento de Historia | La Habana, Cuba

Dr. Rosalva Loreto López, Benemérita Universidad Autónoma de Puebla | Instituto de Cienias Sociales y Humanidades | Puebla - Mexico, México 


\section{is \\ HALAC}

\section{Consejo Científico}

Dr. Andrés Guhl, Universidad de los Andes | Departamento de Historia | Facultad de Ciencias Sociales | Bogotá, Colômbia

Dr. Claudia Leal, Universidad de los Andes | Departamento de Historia | Facultad de Ciencias Sociales | Bogotá, Colômbia

Dr. Eunice Sueli Nodari, Universidade Federal de Santa Catarina (UFSC) | Programa de Pós Graduação em História | Departamento de História | Florianópolis | Santa Catarina, Brasil

Dr. Gabriella Corona, Istituto di Studi sulle Società del Mediterraneo | Napoli, Itália

Dr. Germán Alfonso Palacio Castañeda, Universidad Nacional de Colombia |

Instituto Amazónico de Investigaciones | Letícia | Amazonas, Colômbia

Dr. Guillermo Castro Herrera, Fundación Ciudad del Saber | Ciudad de Panamá, Panamá

Dr. John M Soluri, Carnegie Mellon University | Department of History | Pittsburgh | Pensilvania, Estados Unidos da América do Norte

Dr. Lourdes Elena Lozano Centella, Centro de Incidencia Ambiental de Panamá | Ciudad de Panamá, Panamá

Dr. Mark Carey, University of Oregon | Robert D. Clark Honors College | Eugene | Oregon, Estados Unidos da América do Norte

Dr. Martha Micheline Cariño Olvera, Universidad Autónoma de Baja California Sur | Departamento de Humanidades | La Paz | Baja California Sur, México

Dr. Miguel Aguilar-Robledo, Universidad Autónoma de San Luis Potosí | Coordinación de Ciencias Sociales y Humanidades | San Luis Potosí, México

Dr. Sergio A. Guevara Sada, Instituto de Ecología | Xalapa | Veracruz, México

Dr. Stefania Gallini, Universidad Nacional de Colombia | Departamento de Historia | Bogotá, Colômbia

Dr. Sterling Evans, The University of Oklahoma | Department of History | College of Arts and Sciences | Norman | Oklahoma, Estados Unidos da América do Norte 


\section{is \\ HALAC}

Dr. Stuart McCook, University of Guelph | Department of History | College of Arts | Guelph | Ontario, Canadá

Dr. Teresa Rojas Rabiela, Centro de Investigaciones y Estúdios Superiores en Antropologia Social | Tlalpan | Ciudad de México, México

\section{Editores Técnicos}

Eduardo F. Souza, Portal de Periódicos Eletrônicos da UniEVANGÉLICA, Centro Universitário de Anápolis, Brasil.

Natasha Sophie Pereira, Portal de Periódicos Eletrônicos da UniEVANGÉLICA, Centro Universitário de Anápolis, Brasil.

Josiel Araujo Lemes, Portal de Periódicos Eletrônicos da UniEVANGÉLICA, Centro Universitário de Anápolis, Brasil.

\section{Pareceristas}

Adi Estela Lazos Ruíz, Universidad Nacional Autónoma de México, UNAM, México. Alberto Matarán Ruiz, Universidad de Granada, Espanha.

Ana Marcela França, Universidade Federal do Rio de Janeiro, UFRJ, Brasil.

André Vasques Vital, Centro Universitário de Anápolis, UniEVANGÉLICA, Brasil.

Ayelen Dichdji, Universidad Nacional de Quilmes, Argentina.

Carlos Christian Della Giustina, Centro Universitário de Anápolis, UniEVANGÉLICA, Brasil.

Claiton Marcio da Silva, Universidade Federal da Fronteira Sul, UFFS, Brasil.

Dominichi Miranda de Sá, Fundação Oswaldo Cruz, FIOCRUZ, Brasil.

Dora Shellard Correa, Centro Universitário da FIEO, Brasil.

Euripedes Funes, Universidade Federal do Ceará, UFC, Brasil.

Hamilton Afonso de Oliveira, Universidade Estadual de Goiás, UEG, Brasil. 


\section{is \\ HALAC}

John M. Soluri, Carnegie Mellon University, CMU, United States.

José Andrés Ibáñez de Miguel, University of Granada, Espanha.

José Luiz Andrade Franco, Universidade de Brasília, UnB, Brasil.

Luciana Murari, Universidade Federal de Minas Gerais, UFMG, Brasil.

María del Carmen Villarreal Villamar, Universidade Federal do Estado do Rio de Janeiro, UNIRIO, Brasil.

Marina Miraglia, Universidad Nacional de General Sarmiento, UNGS, Brasil.

Matthew Vitz, Friedrich Schiller University Jena, FSU, Germany.

Myrna Santiago, Saint Mary's College of California, Estados Unidos.

Paulo Henrique Martinez, Universidade Estadual Paulista Júlio de Mesquita Filho, UNESP, Brasil.

Pedro Pietrafesa, Pontifícia Universidade Católica de Goiás (PUC Goiás), Brasil.

Roger Domenech Colacios, Universidade Estadual de Maringá, UEM, Brasil.

Samira Peruchi Moretto, Universidade Federal da Fronteira Sul, Brasil.

Sílvio Marcus de Souza Correa, Universidade Federal de Santa Catarina, UFSC, Brasil.

Sônia Maria de Magalhães, Universidade Federal de Goiás, UFG, Brasil.

Vladimir Sánchez Calderón, Universidad Industrial de Santander, Colômbia.

Wilson Picado, National University of Costa Rica, UMA, Costa Rica. 


\section{is \\ HALAC}

Editorial

Carta Editorial: Balances de Historia Ambiental en América Latina

Sandro Dutra e Silva, Marina Miraglia, Wilson Picado

09-15

\section{Artículos}

História dos animais no Brasil: tradições culturais, historiografia e transformação.

Regina Horta Duarte

Sociedades, ambiente y ambientalismos en nuestra América

Guillermo Castro Herrera

Considerações ético-políticas na História (Ambiental): escalas e o presentismo da devastação

Gilmar Arruda

Irrigación y Organización Social en una Sociedad en Transición al Capitalismo: el Caso de la Asociación de Canalistas del Maipo en Chile (S.XIX)

Pablo Camus; Guillermo Elgueda; Enrique Muñoz

Para plantar e colher arroz é preciso redesenhar a paisagem:

narrativas de imigrantes europeus sobre o cultivo do arroz na

Colônia Dona Francisca (século XIX)

Alanna Fernandes Duarte; Mariluci Neis Carelli;Roberta Barros Meira

Explorar, contar, proteger. La emergencia de los bosques como problema ambiental en la historia argentina

María Paula Avila Castro

Derrubar ou conservar as matas reais: o debate sobre as políticas 


\section{is \\ HALAC}

florestais na Capitania do Ceará (1797-1806).

Diego Estevam Cavalcante

Violencia y Extractivismo en el Perú contemporâneo

$210-236$

Raquel Viviana Neyra Soupplet

Da Serra dos Cavalos ao Vale do Ipojuca (Caruaru/PE): águas e história ambiental no semiárido brasileiro

$237-262$

João Domingos Pinheiro Filho

\section{Reseñas}

El Agua de la Revolución: los claroscuros de la modernidad tecnoambiental mexicana

Julio Aguilar Hidalgo

A Grande transformação que vinha da colonização dirigida

Henrique Dias Sobral Silva

La relación dialéctica entre formación del Estado y conservación de la naturaliza

Olaf Kaltmeier

Para nos Contatar: Revista HALAC - Portal de Periódicos Eletrônicos UniEVANGÉLICA, Avenida Universitária Km 3,5 Cidade Universitária, CEP: 7583515. Anápolis, Goiás, Brasil.

Telefone: +55 06233106679

solcha.halac@gmail.com・https://www.halacsolcha.org/

Capa: Guanacaste, Costa Rica. 2019.

Fotografia: Wilson Picado.

Projeto Gráfico e Editoração: E. F. Souza, J; A. Lemes 2019. 